\title{
Comparing the Efficacy of a Nurse-Driven and a Physician-Driven Diabetic Ketoacidosis (DKA) Treatment Protocol
}

\author{
Takla R Anis' \\ Marybeth Boudreau' \\ Tyson Thornton ${ }^{2}$ \\ 'Pharmacy Department, Northern Light \\ Eastern Maine Medical Center, Bangor, \\ ME, USA; ${ }^{2}$ Pharmacy Department, \\ Northern Light Sebasticook Valley \\ Hospital, Pittsfield, ME, USA
}

Background: Standardized DKA treatment could result in better overall safety and efficacy outcomes. The primary objective of this study is to validate the efficacy of an adapted nursedriven DKA protocol compared to a physician-driven DKA protocol across the continuum of three hospital settings: the University of Colorado upon which the physician-driven protocol is based, Northern Light Eastern Maine Medical Center (NLEMMC), and Northern Light Sebasticook Valley Hospital (NLSVH). The secondary objective is to assess the safety of the adapted nurse-driven DKA protocol adapted at NLEMMC and NLSVH through determining the incidence of hypoglycemia and anion gap reopening.

Patients and Methods: This was a retrospective, IRB-approved, multi-center study that included: patients 18 years or older who were treated with the DKA protocol at NLEMMC or NLSVH, and admitted to the emergency department between July 2015 and October 2020 with a primary diagnosis of DKA and an elevated anion gap greater than or equal to $13 \mathrm{mEq} / \mathrm{L}$.

Results: A total of 90 patients from NLEMMC and 64 patients from NLSVH were included and compared to 111 patients from the University of Colorado who were included in the post protocol implementation group. There was no statistically significant difference in the primary outcome, time to anion gap closure, between the original University of Colorado study (10.3 hours) and the NLEMMC (10.9 hours, $p=0.420)$ and NLSVH (8.8 hours, $p=$ $0.115)$ results presented in this study.

Conclusion: The standardized nurse-driven DKA treatment protocol at NLEMMC and NLSVH showed no statistical difference in time to anion gap closure compared to the University of Colorado study upon which it was based. This finding is particularly relevant to hospitals such as NLEMMC and NLSVH that lack provider resources and teams of endocrinologists required for the physician-driven DKA protocol.

Keywords: diabetic ketoacidosis, protocol, treatment

\section{Introduction}

Diabetic ketoacidosis (DKA) is one of the most severe complications that can affect diabetic patients. Along with the detrimental health consequences, DKA accounts for over 500,000 hospital days per year with an annual total cost of about 2.4 billion USD. ${ }^{1-3}$ There are three main complications that DKA patients suffer from and typically present with: dehydration, hyperglycemia, and electrolyte imbalances. Standardized treatment of DKA can result in faster resolution of the disease state by correcting these complications in a timely fashion. Additionally, implementing ordersets and treatment protocols can prevent inappropriate subcutaneous insulin administration and potential confusion
Correspondence: Takla R Anis Pharmacy Department, Northern Light Eastern Maine Medical Center, Bangor, ME, 0440I, USA

Email takla.anis@gmail.com 
amongst care team members. In their retrospective chart review, Donihi et al demonstrated the impact of utilizing a DKA orderset as more patients received subcutaneous insulin in a timely fashion ensuring appropriate overlap with insulin infusions where appropriate. ${ }^{4}$ In 2007, Bull et al from the University of Colorado and Health Sciences Center published a mandatory physician-driven protocol for treating adult patients with DKA, which showed a quick resolution of DKA and significant reductions in intensive care unit and hospital lengths of stay. ${ }^{5}$ Unlike the University of Colorado, the Northern Light Health System Hospitals do not always have the luxury of physician resources and endocrinology teams. In 2010, Northern Light's tertiary care facility, Northern Light Eastern Maine Medical Center (NLEMMC) adapted Bull et al's physician-driven protocol and implemented it into a nurse-driven DKA protocol. ${ }^{5}$ Since 2010, this nurse-driven DKA protocol has been adapted and utilized across all of the Northern Light Health system institutions, including Sebasticook Valley Hospital (NLSVH), a 25-bed critical access hospital (CAH). NLEMMC and NLSVH admit approximately 90 and 16 DKA patients, respectively, per year. In this study, we attempted to determine the efficacy and safety of our adapted nurse-driven protocol in comparison to the Bull et al study across the spectrum of two very different hospital entities. If proven to be equally safe and effective, our adapted nurse-driven protocol can be utilized by other institutions across the country, especially those in rural and criticalaccess hospitals lacking provider resources.

\section{Materials and Methods Study Design and Outcomes}

This was a retrospective, multicenter observational cohort trial. The study was approved by all institutional review boards at Northern Light Health System Hospitals (NLH). Data were collected through chart review. The primary outcome was time to resolution of DKA defined as the time between the first elevated anion gap value identified in the emergency department and the first anion gap value less than $13 \mathrm{mEq} / \mathrm{L}$. Time to anion gap closure at each hospital was compared to the time until anion gap closure presented in the original Bull et al. study. ${ }^{5}$ Secondary outcomes included hospital length of stay, recurrence of anion gap opening, and incidence of hypoglycemia.

\section{Participants}

In this study, we included adult patients 18 years of age or older who had orders for the established NLH DKA protocol at NLEMMC or NLSVH, and were admitted to the emergency department between July 2015 to October 2020 with a primary diagnosis of DKA and an elevated anion gap greater than $13 \mathrm{mEq} / \mathrm{L}$. We utilized a similar DKA diagnosis criteria to what was highlighted in the Bull et al. study. ${ }^{5}$ Patients were excluded if: (1) they had more than 2 deviations from our DKA protocol (ie, change in fluid selection or an insulin dose administered outside of the protocol), (2) pregnant, (3) imprisoned, (4) transferred from an outside facility where treatment was initiated for DKA, or (5) were admitted with a concurrent diagnosis of sepsis or respiratory failure.

\section{Data Collection}

Patients with multiple admissions for DKA were included only once in the study, which was their initial admission. The following patient information and pertinent lab values were obtained from the electronic medical records of both institutions: age, gender, weight, creatinine clearance, emergency department admission date and time, initial anion gap date and time, initial $\mathrm{Ph}$, initial potassium level, initial blood glucose (BG) level, initial heart rate (HR), initial mean arterial pressure (MAP), diabetes type, date and time of anion gap (AG) closure, ICU admission date and time, ICU discharge date and time, and hospital discharge date and time. We also collected date and time of anion gap reopening within 6 hours from the initial closure to further validate the efficacy of our protocol. ICU and hospital length of stay were calculated from the collected data.

\section{Statistical Analyses}

The primary outcome for this study was time to anion gap closure. An a priori power analysis was performed for a twosided two-sample $t$-test, which identified a minimum of 55 patients were needed to attain an $80 \%$ power assuming a statistical significance $\alpha=0.05$, a 4.6-hour standard deviation (cf. Bull et al), and a hypothesized time difference of + 2.5 hours from Bull et al's 10.3-hour stated mean time to anion gap closure. ${ }^{5}$ Due to the inability to obtain raw data from the original study, two-sample $t$-tests of summarized data were utilized to compare non-proportional data (eg, age, initial blood glucose (BG), time to anion gap (AG) closure) between the University of Colorado hospital and each of the two hospitals in this study. Two-sample proportion hypothesis tests assuming normal approximations were utilized to determine statistical significance for proportional data (eg, male sex, AG reoccurrence, incidence of hypoglycemia). All statistical analyses were performed in Minitab 19. 


\section{Results}

A total of 209 patients aged 18 years old or older having initial diagnoses of diabetic ketoacidosis were initially screened from patients admitted between July 2015 and October 2020 at NLEMMC and NLSVH hospitals. Inclusion criteria were met in 90 patients from NLEMMC and 64 patients from NLSVH. Patients were mainly excluded for being transferred from outside facilities where DKA treatment was initiated and for having concurrent diagnosis of sepsis or respiratory failure. Patient demographics data and pertinent lab values are summarized in Table 1, where both hospitals are compared with the original study from the University of Colorado. As shown in Table 2, there was no statistically significant difference in the primary outcome time to anion gap closure between NLEMMC and the University of Colorado, and NLSVH and the University of Colorado. Although the secondary outcomes (ie, hospital length of stay, AG reoccurrence, and incidence of hypoglycemia) were generally similar between the University of Colorado and NLEMMC and NLSVH, there were three statistically significant differences. First, the hospital length of stay for NLSVH was significantly shorter than the length of stay for the University of Colorado. Second, the AG reoccurrence for NLEMMC was significantly greater than AG reoccurrence for the University of Colorado. Third, the incidence of hypoglycemia for NLSVH was significantly less than the incidence of hypoglycemia for the University of Colorado.

\section{Discussion}

Our study showed the impact of utilizing ordersets to standardize treatment pathways in high-risk conditions like DKA, where time to resolution of the disease state is a major component to preventing further complications. Compared to results from Bull et al for the University of Colorado, ${ }^{5}$ our protocol demonstrated similar outcomes and required fewer provider resources. Thus, the nurse-driven protocol presented here could lead to reductions in the overall treatment costs for DKA due to reduced length of stay and required resources while producing similar patient outcomes. Unlike the mandatory physician-driven protocol disclosed in Bull et al, our protocol is completely nurse-driven. In 2010, the pharmacy department along with a group of intensivists from the NLH System Hospitals were able to build a DKA

Table I Patient Demographics

\begin{tabular}{|c|c|c|c|c|c|}
\hline & $\begin{array}{l}\text { University of Colorado } \\
\qquad(n=\mid I I)\end{array}$ & $\begin{array}{c}\text { NLEMMC } \\
(\mathrm{n}=90)\end{array}$ & $p$ value & $\begin{array}{l}\text { NLSVH } \\
(n=64)\end{array}$ & $p$ value \\
\hline Age, yrs (mean $\pm S D$ ) & $39 \pm 13$ & $42.7 \pm 17.9$ & 0.103 & $47.6 \pm 15.5$ & 0.001 \\
\hline Male sex, $\%$ & 63 & 59 & 0.546 & 41 & 0.003 \\
\hline Initial BG (mean $\pm S D)$ & $588 \pm 245$ & $555.9 \pm 230.9$ & $0.34 I$ & $530.4 \pm 231.7$ & 0.123 \\
\hline Initial HR (mean $\pm S D$ ) & $108 \pm 19$ & $105.9 \pm 18.7$ & 0.433 & $107 \pm 19.9$ & 0.745 \\
\hline Initial MAP (mean $\pm S D)$ & $105 \pm 17$ & $92.3 \pm 16.2$ & $<0.001$ & $93.2 \pm 16.7$ & $<0.001$ \\
\hline Diabetes type (pts) & - & $\begin{array}{c}\text { Type I } \rightarrow 62 \\
\text { Type II } \rightarrow 18 \\
\text { Unknown } \rightarrow 10\end{array}$ & - & $\begin{array}{c}\text { Type I } \rightarrow 29 \\
\text { Type II } \rightarrow 3 \text { I } \\
\text { Unknown } \rightarrow 4\end{array}$ & - \\
\hline
\end{tabular}

Table 2 Outcomes

\begin{tabular}{|l|c|c|c|c|c|}
\hline Outcome & $\begin{array}{c}\text { University of } \\
\text { Colorado } \\
(\mathbf{n}=1 \mathrm{II})\end{array}$ & $\begin{array}{c}\text { NLEMMC } \\
(\mathbf{n}=\mathbf{9 0})\end{array}$ & $\boldsymbol{P}$ value & $\begin{array}{l}\text { NLSVH } \\
(\mathbf{n}=\mathbf{6 4})\end{array}$ & $\mathbf{p}$ value \\
\hline Time to AG closure (hrs) & $10.3 \pm 4.6$ & $10.9 \pm 5.7$ & 0.420 & $8.8 \pm 6.8$ & 0.120 \\
\hline Hospital length of stay (hrs) & $64.3 \pm 41.4$ & $68.1 \pm 50.6$ & 0.567 & $49.6 \pm 34.0$ & 0.012 \\
\hline AG reoccurrence (>I5 meq/L) & $3 \%(3 \mathrm{pts})$ & $11.1 \%(10 \mathrm{pts})$ & 0.021 & $3.1 \%(2 \mathrm{pts})$ & 0.874 \\
\hline Incidence of Hypoglycemia (BG <55) & $14 \%(18 \mathrm{pts})$ & $18.9 \%(17 \mathrm{pts})$ & 0.621 & $4.7 \%(3 \mathrm{pts})$ & 0.009 \\
\hline
\end{tabular}


calculator as part of the DKA orderset based on the titration table from the original study. ${ }^{5}$ This calculator directed nurses to administer and adjust the rates of insulin and fluids based on the patient's lab values. This demonstrates the feasibility of our protocol especially at a small 25-bed critical access hospital like NLSVH, where providers sometimes manage patients remotely. This allows patients to receive care close to home and limits the need for patient transfers to larger facilities where bed capacity can be limited. DKA is a complicated disease state that requires the medical team to consider multiple factors. As highlighted by Bull et al, one of the major advantages of this protocol is that it provides clear treatment pathways for essential therapy components like adequate fluid resuscitation, electrolyte replacement, and appropriate insulin dosing. ${ }^{5}$ Despite being more aggressive compared to other published protocols, our protocol did not result in significant adverse effects, as evidenced by the incidence of hypoglycemia results (NLEMMC 18.9\% and NLSVH 4.6\%). In 2006, the American College of Endocrinology and American Diabetes Association released a consensus statement that highlighted some of the main challenges in managing hyperglycemic patients, which included a national shortage in nurses. ${ }^{6}$ Furthermore, this could lead to hesitancy and inconsistency in insulin administration due to the fear of causing hypoglycemia. ${ }^{6}$ One of the main challenges we face with utilizing the protocol is providing continuous education to the interdisciplinary team members regarding coadministration of long-acting subcutaneous insulin and overlapping insulin infusion. In order to ensure a safe and effective application of our protocol, we provided intense, on-going education to our ICU and emergency teams regarding when to initiate the protocol, how to order labs through the DKA orderset, and when to notify the provider in severe cases in which the patient is non-responsive to therapy. One of the potential causes that led to a significantly higher incidence of gap re-opening at NLEMMC is a high turnover rate for ICU nurses, which could have led to unintentional non-adherence to the protocol. This issue was previously highlighted at other large institutions resulting in prolonged treatment times to resolution of the disease state. For instance, in her retrospective chart review, Ruth Ferreri found that the majority of patients did not receive appropriate overlap between continuous insulin infusions and longacting subcutaneous insulin. ${ }^{7}$ One of the interesting findings in our study is the difference in patient populations between NLEMMC and NLSVH. For instance, more patients with type 1 diabetes were included from NLEMMC (69\%) compared to NLSVH (45\%). However, there was no clinically significant difference in time to anion gap closure, which aligns with the findings from a multicenter study by Balmier et al who also did not find a significant difference in time to recovery between type 1 and type 2 diabetic patients. ${ }^{8}$ On the other hand, Balmier et al highlighted an interesting finding as they found a significant difference in metabolic complications like hypoglycemia and hypokalemia associated with DKA treatment depending on the underlying type of diabetes. ${ }^{8}$ This issue is not clearly highlighted by the current treatment guidelines and protocols, which will need further studies to investigate the difference in insulin requirements depending on the underlying diabetes type to avoid potential future treatment complications. It is also unclear whether concurrent disease states could have an impact on DKA patients depending on their underlying diabetes type. In their retrospective cohort study, Kempegowda et al highlighted findings indicating COVID-19 impacts DKA differently in type 1 and type 2 diabetics. ${ }^{9}$ Furthermore, type 2 diabetics required greater ICU length of stay and had a higher mortality rate compared to type 1 diabetics who were more hyperglycemic upon presentation. ${ }^{9}$ Our study did not include any COVID-19 patients since our enrollment period was between July 2015 to October 2020. Given the increasing incidence of COVID-19, larger prospective multicenter studies are needed to determine if there are any treatment adjustments that need to be made when treating DKA patients with concurrent diagnosis of COVID-19. Additionally, as highlighted by Islam et al, further updates are required to the current treatment DKA protocols and guidelines to ensure their appropriateness when utilizing them for other special patient populations like heart failure and renally impaired patients. ${ }^{10}$ As shown in the Supplementary Appendix, our DKA treatment protocol recommends adjusting both total fluid volumes and rates of administration for patients with heart failure, end-stage liver or renal disease, those over age 65 , or hypoxemia to prevent fluid overload.

\section{Limitations}

The study was affected by at least three limitations. The first limitation of this study was a small sample size which could have resulted from the strict exclusion criteria as many patients were excluded as a result of being transferred in from outside hospitals where DKA treatment was initiated and was not in adherence with the NLH protocol. This approach was taken to ensure accurate assessment of our DKA protocol. Although the sample size $n=55$ with a hypothesized time difference of \pm 2.5 hours for a 4.6-hour 
standard deviation resulted in an $80 \%$ power, the actual time differences and standard deviations for time to $\mathrm{AG}$ closure were 0.6 and 5.7 hours, respectively, for NLEMMC, and 1.5 and 6.8 hours, respectively, for NLSVH. An a posteriori power and sample size calculation indicates that the 90patient sample size having a 5.7-hour standard deviation as found for NLEMMC can detect a \pm 2.4-hour time difference at an $80 \%$ power. Similarly, the 64 -patient sample size having a 6.8-hour standard deviation as found for NLSVH can detect $\mathrm{a} \pm$ 3.4-hour time difference at an $80 \%$ power. Although we were unable to achieve our statistical targets, our protocol demonstrated clinical success and efficacy in real-world practice. The second limitation was the lack of documentation of certain lab values like initial $\mathrm{pH}$, which made it difficult to accurately assess the severity of illness as we were unable to calculate accurate APACHE II scores for many of our patients. The third limitation was that NLSVH is a small, critical access hospital that lacks transitions of care. Patients who get admitted with multiple acute conditions and require higher level of care get transferred to higher level of care at other hospitals like NLEMMC. Hence, some of the secondary outcomes, such as hospital length of stay between NLSVH and the University of Colorado is not an accurate representation of the overall hospital length of stay because of the difference in patient population and hospital settings. However, from a practical standpoint, the overall hospital length of stay at NLSVH was 48.9 hours, which ensures that patients receive appropriate treatment in a timely manner at a relatively smaller hospital with limited bed-space capacity and provider resources. Given the overall lack of healthcare resources in rural communities, the benefits of being able to treat complex patients with a protocol-based approach, like those in DKA, at rural care centers cannot be overlooked.

\section{Conclusion}

Our mandatory nurse-driven DKA protocol demonstrated equivalent efficacy and safety compared to the original physician-driven DKA protocol upon which it was based. ${ }^{5}$ Standardized DKA treatment could ultimately lead to better results when compared to individualized treatment. Future studies will need to be conducted prospectively across similar hospital settings with similar patient populations to further assess the utility of implementing new strategies that could result in faster resolution of the disease state with additional consideration being given to concomitant disease states on patient presentation. Also, more studies are needed to further investigate the overall cost reduction and overall benefits to the healthcare system like re- admission rates associated with using standardized DKA treatment protocols compared to individualized treatment.

\section{Consent}

Northern Light Eastern Maine Medical Center's institutional review board (IRB) approval was obtained prior to conducting the study, which included all consent forms. This was an informational retrospective study that was given an exempt status for obtaining patient consent. This study was conducted in accordance with the Declaration of Helsinki.

\section{Acknowledgments}

We would like to acknowledge Dr. Sarah V Bull and colleagues from the University of Colorado and Health Sciences Center.

\section{Disclosure}

The authors report no conflicts of interest in this work.

\section{References}

1. Kitabchi AE, Umpierrez GE, Miles JM, Fisher JN. Hyperglycemic crises in adult patients with diabetes. Diabetes Care. 2009;32 (7):1335-1343. doi:10.2337/dc09-9032

2. Kim S. Burden of hospitalizations primarily due to uncontrolled diabetes: implications of inadequate primary health care in the United States. Diabetes Care. 2007;30:1281-1282. doi:10.2337/ dc06-2070

3. National Center for Health Statistics. National hospital discharge and ambulatory surgery data [article online]. Available from: http://www. cdc.gov/nchs/about/major/hdasd/nhds.htm. Accessed January 24, 2009.

4. Donihi A, Karajgikar ND, Manroa P, et al. Addressing pitfalls in management of diabetic ketoacidosis with a standardized protocol. Endocr Pract. 2019;25(5):407-412. doi:10.4158/EP-2018-0398

5. Bull SV, Douglas IS, Foster M MT(ASCP), Albert RK MD. Mandatory protocol for treating adult patients with diabetic ketoacidosis decreases intensive care unit and hospital lengths of stay: results of a nonrandomized trial. Crit Care Med. 2007;35(1):41-46. doi:10.1097/01.CCM.0000249825.18677.D2

6. ACE/ADA Task Force on Inpatient Diabetes. American college of endocrinology and American diabetes association consensus statement on inpatient diabetes and glycemic control. Diabetes Care. 2006;29(8):1955-1962. PMID: 16873812. doi:10.2337/dc06-9913

7. Ferreri R. Treatment practices of diabetic ketoacidosis at a large teaching hospital. J Nurs Care Qual. 2008;23(2):147-154. PMID: 18344781. doi:10.1097/01.NCQ.0000313764.64149.6d

8. Balmier A, Dib F, Serret-Larmande A, et al. Initial management of diabetic ketoacidosis and prognosis according to diabetes type: a French multicentre observational retrospective study. Ann Intensive Care. 2019;9:91. doi:10.1186/s13613-019-0567-y

9. Kempegowda P, Melson E, Johnson A, et al. Effect of COVID-19 on the clinical course of diabetic ketoacidosis (DKA) in people with type 1 and type 2 diabetes. Endocr Connect. 2021;10(4):371-377. doi:10.1530/EC20-0567

10. Islam T, Sherani K, Surani S, Vakil A. Guidelines and controversies in the management of diabetic ketoacidosis - a mini-review. World $J$ Diabetes. 2018;9(12):226-229. PMID: 30588284; PMCID: PMC6304293. doi:10.4239/wjd.v9.i12.226 


\section{Publish your work in this journal}

Clinical Pharmacology: Advances and Applications is an international, peer-reviewed, open access journal publishing original research, reports, reviews and commentaries on all areas of drug experience in humans. The manuscript management system is completely online and

Submit your manuscript here: https://www.dovepress.com/clinical-pharmacology-advances-and-applications-journal includes a very quick and fair peer-review system, which is all easy to use. Visit http://www.dovepress.com/testimonials.php to read real quotes from published authors. 